\title{
Multimode trapped interferometer with noninteracting Bose-Einstein condensates
}

\author{
Leonardo Masi, ${ }^{1,2}$ Tommaso Petrucciani, ${ }^{2,3}$ Alessia Burchianti $\odot,{ }^{1,2}$ Chiara Fort $\odot,{ }^{1,2,3}$ Massimo Inguscio, ${ }^{1,2,4}$ \\ Lorenzo Marconi $\odot,{ }^{1}$ Giovanni Modugno $\odot,{ }^{1,2,3}$ Niccolò Preti, ${ }^{3}$ Dimitrios Trypogeorgos, ${ }^{5}$ \\ Marco Fattori $\odot,{ }^{1,2,3, *}$ and Francesco Minardi ${ }^{1,2,6}$ \\ ${ }^{1}$ CNR-INO, Istituto Nazionale di Ottica, 50019 Sesto Fiorentino, Italy \\ ${ }^{2}$ European Laboratory for Nonlinear Spectroscopy-LENS, 50019 Sesto Fiorentino, Italy \\ ${ }^{3}$ Dipartimento di Fisica e Astronomia, Università di Firenze, 50019 Sesto Fiorentino, Italy \\ ${ }^{4}$ Dipartimento di Ingegneria, Campus Biomedico Università di Roma, 00128 Roma, Italy \\ ${ }^{5}$ CNR Nanotec, Institute of Nanotechnology, 73100 Lecce, Italy \\ ${ }^{6}$ Dipartimento di Fisica e Astronomia, Università di Bologna, 40127 Bologna, Italy
}

(Received 25 May 2021; accepted 27 October 2021; published 17 December 2021)

\begin{abstract}
We experimentally demonstrate a multimode interferometer comprising a Bose-Einstein condensate of ${ }^{39} \mathrm{~K}$ atoms trapped in a harmonic potential, where the interatomic interaction can be canceled exploiting Feshbach resonances. Kapitza-Dirac diffraction from an optical lattice coherently splits the BEC in multiple momentum components equally spaced that form different interferometric paths, with trajectories closed by the trapping harmonic potential. We investigate two different interferometric schemes, where the recombination pulse is applied after a full or half oscillation in the confining potential. We find that the relative amplitudes of the momentum components at the interferometer output are sensitive to external forces, through the induced displacement of the harmonic potential with respect to the optical lattice. We show how to calibrate the interferometer, fully characterize its output, and discuss perspective improvements.
\end{abstract}

DOI: 10.1103/PhysRevResearch.3.043188

\section{INTRODUCTION}

Developed over three decades, atom interferometry represents the state of the art for the measurements of accelerations $[1,2]$ and rotations [3] with unparalleled resolution, for the precise determination of fundamental constants [4-6], and for testing the equivalence principle $[7,8]$. In these interferometers, atomic samples are split into two separate paths and recombined by multiple photon transitions. Since most of those experiments are based on free-falling atomic samples, the resolution typically scales as the square of the interrogation time, i.e., linearly with the length of the interferometer. However, on one hand, lengthy interferometers are technically demanding, since they require managing the spread of atomic wave packets and controlling external perturbations over large regions of space; on the other hand, their spatial resolution is obviously limited.

Interferometry with trapped atoms offers the distinct advantage of extended interrogation times in compact setups, measuring forces and local fields with spatial resolution of a few micrometers [9]. Several experiments have been performed using Bose-Einstein condensates (BECs) trapped in

\footnotetext{
*Corresponding author: fattori@lens.unifi.it

Published by the American Physical Society under the terms of the Creative Commons Attribution 4.0 International license. Further distribution of this work must maintain attribution to the author(s) and the published article's title, journal citation, and DOI.
}

magnetic traps $[10,11]$, generated near the surface of an atom chip [12], and in optical double-well potentials $[13,14]$. In these works the main effort was directed toward engineering the external potential used to coherently split and recombine the wave function into two spatially separated modes. In addition to exploiting long interrogation times, trapped atom interferometers can increase the interferometric phase in a direct way by enlarging the spatial separations between the paths. This goal has been pursued by different methods, e.g., by implementing double-well potentials with separations of several microns [14], by coupling Wannier-Stark states that are several lattices sites distant [15], by holding the two paths in a vertical lattice separated by large distances [16], or by splitting a single condensate in the two traps formed by a transverse mode of an optical cavity [17]. A recent proposal has also suggested to exploit the spread of the wave function during the Bloch dynamics in a horizontal lattice in the presence of a weak force as a simple way to increase the spatial separations of the atoms and consequently increase the sensitivity of the interferometer [18].

The present work demonstrates an interferometric method for trapped quantum gases based on a multimode configuration, with more than two interferometric paths, where the coherent splitting and recombination of a BEC into multiple momentum components are realized by means of KapitzaDirac (KD) diffraction from a pulsed optical lattice. With this configuration, proposed theoretically for noninteracting systems by Li et al. [19], the harmonic potential forgoes the need of the "mirror" light pulses to close the trajectories and prevents the spatial spread of wave packets, instead occurring 
in free space. KD interferometers have been implemented experimentally either in the Mach-Zehnder two-mode configuration in a waveguide [20], or in trap [21], still with only two interfering modes and with ${ }^{87} \mathrm{Rb}$ atoms for which the interpretation of the interference fringes is complicated by the interatomic interactions [22]. Like in the above-mentioned interferometers, also our sensitivity on the measured acceleration increases with the spatial separation of the paths; it reaches a level allowing the detection of the beam-pointing instability of the optical trap.

More specifically, we report here on the realization of a horizontal multimode trapped interferometer, where a noninteracting Bose-Einstein condensate is KD diffracted by an optical lattice into components with momenta equal to multiples of the lattice wave vector $k$, i.e., with $p=m \hbar k$, with $m$ integer. A KD pulse initiates the oscillation of the different momentum orders which, after half an oscillation in the harmonic trap, return to the initial position with opposite momenta and are recombined using another KD pulse. Importantly, all the momentum components spatially recombine at the trap minimum only if the potential is harmonic over their oscillation amplitude. This is easily the case for magnetic traps generated by macroscopic coils [21], but it represents a tight constraint for optical dipole traps (ODTs), that are the most common choice when, e.g., the control of interactions through Feshbach resonances is sought. Here we use an optical trap and take advantage of a large-spacing $(\sim 5 \mu \mathrm{m})$ optical lattice, that reduces the recoil velocity, hence the oscillation amplitude, by a factor of 10 , with respect to the commonplace lattice spacing of $0.5 \mu \mathrm{m}$. Specifically, we create the periodic potential exploiting a recently developed technique, named the "beat-note superlattice" [23], capable of realizing lattices with a large effective period in a retroreflected configuration, with laser wavelengths of the order of $1 \mu \mathrm{m}$. In order to investigate experimentally the operation of the KD interferometer in the presence of external perturbations, we first observe the evolution of the different diffracted orders in the ODT, confirming a harmonic and symmetric evolution. Then, we calibrate the output distribution as a function of the time interval and the phase difference between the two pulses after a full oscillation. These measurements serve to set the right parameters during the operation. We then apply a controlled horizontal force through a magnetic field gradient and measure the momentum distribution after half an oscillation, for different values of the gradient field. We observe a clear dependence of the atomic populations in the different momentum components on the external force and we compare the observed results with analytical predictions.

\section{THEORETICAL ANALYSIS}

A KD interferometer in a harmonic trap of frequency $\omega$ detects an unknown acceleration $a$ through the induced displacement of the trap minimum, $d=a / \omega^{2}$, measured with respect to the wave front of the applied optical lattice. The displacement is encoded in the phase factor $e^{i m \phi}$, with $\phi=k d$, imprinted by the KD pulses on the different momentum components $m \hbar k$.

The basic working principle of the interferometer can be best visualized as follows: a KD pulse generates three mo-

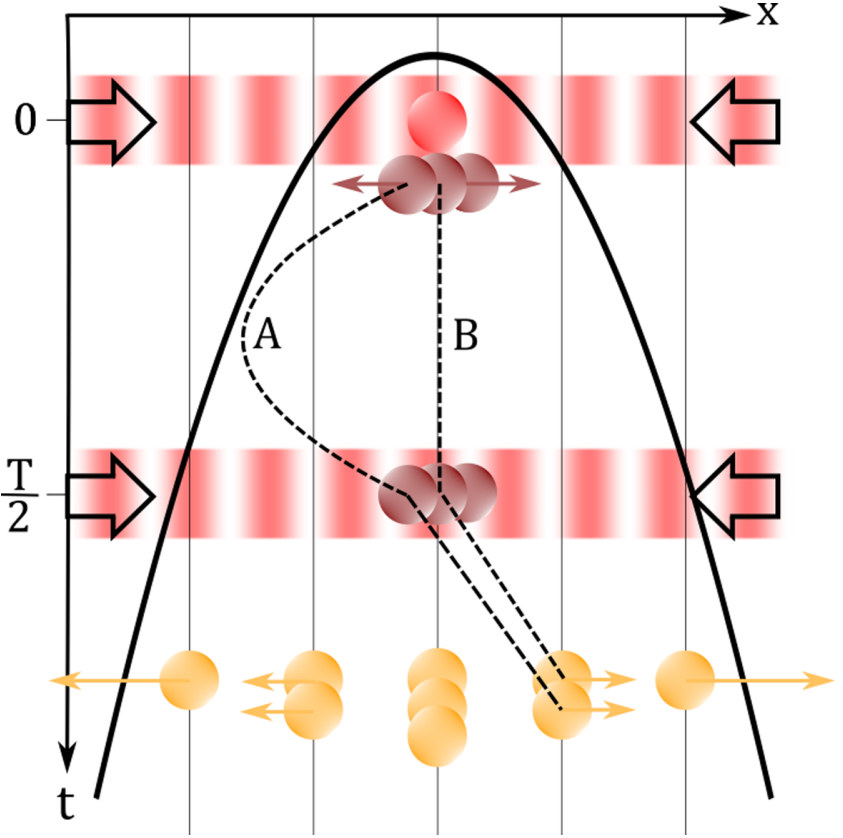

FIG. 1. Sketch of the basic working of our trapped Kapitza-Dirac interferometer. Starting from a single BEC (red), two KD pulses at $t=0$ and $T / 2$ create and recombine the momentum components that oscillate in the trap (purple) and are finally detected after a free expansion (yellow). Colored arrows, indicating the momentum vectors, are not shown at $t=T / 2$ for clarity. We highlight the two different paths $\mathrm{A}$ and $\mathrm{B}$ as identified in the text, from which the $m=+1$ component emerges at the output of the interferometer.

mentum components $m=0, \pm 1$; after this pulse, the half oscillation in the trap reverses the momentum of all components, before the second KD pulse further splits each in three. Thus, the $m=+1$ component emerges from the second KD pulse through two distinct paths, as illustrated in Fig. 1: (A) the $m=-1$ component is generated by the first KD pulse, its momentum is reversed into $m=+1$ by the half-period evolution, and then it is left unperturbed by the second KD pulse; (B) the $m=0$ component emerging from the first KD pulse is scattered into $m=+1$ by the second. On path A the phase factor $e^{-i \phi}$ is imprinted by the first KD pulse, while on path $\mathrm{B} e^{+i \phi}$ is imprinted by the second KD pulse: their sum produces interference on the momentum population at the interferometer output.

Indeed, like in any other light-pulse interferometer [24] the interferometric phase accumulated on each path is obtained as the sum of the terms originated during the light-atom interaction and terms due to the evolution between the light pulses. The latter are given by the action along the classical trajectory, which vanishes whenever the time separation between the two light pulses equals an integer multiple of the harmonic half period, independently of the initial momentum.

Taking into account all the momentum components, the wave function at the interferometer output is exactly calculated [19]. We consider that the initial wave function $\psi_{0}(x)$ corresponds to the ground state of the harmonic oscillator, that the lattice potential during the two KD pulses is given by $V_{i}(x)=V_{0} \sin \left(k x+\phi_{i}\right)(i=1,2)$, and the pulse duration $\delta t$ is so short that only the wave function phase is affected (Raman- 
Nath limit [25]). Then, the wave function at the interferometer output after half oscillation is

$$
\begin{aligned}
\psi_{H}(x) & =\psi_{0}(-x) e^{-i 2 \beta \sin \phi \cos (k x+\delta)} \\
& =\psi_{0}(-x) \sum_{m} J_{m}(2 \beta \sin \phi)(-i)^{m} e^{i m k x} e^{i m \delta},
\end{aligned}
$$

where $\beta=V_{0} \delta t / \hbar, \phi=\left(\phi_{1}+\phi_{2}\right) / 2$, and $\delta=\left(\phi_{2}-\phi_{1}\right) / 2$ are the average and semidifference of the phases of the two pulses, and $J_{m}$ denotes the Bessel function of order $m$. Clearly a displacement $d$ of the harmonic potential is equivalent to a translation of both phases by $k d$.

The acceleration, that gives rise to the displacement $d$, can be measured considering various observables constructed from the output populations. One notable example, on which we will focus in the following, is the fraction of atoms remaining in the initial $m=0$ component, $O_{1}=N_{0} / N$ : due to the multimode interference, varying $d$ this observable is expected to display a peak that narrows as the number of interfering components increases. The half-width at half-maximum (HWHM) phase, corresponding to $J_{0}^{2}\left(2 \beta \sin \phi_{\mathrm{HWHM}}\right)=1 / 2$, is $\phi_{\mathrm{HWHM}}=k d_{\mathrm{HWHM}} \simeq 0.56 / \beta$. This is similar to light interference with multiple beams occurring, e.g., in a high-finesse optical cavity: in the very same way, the resolution of the KD interferometer increases with $\beta$ at the expense of its dynamic range [19]. More specifically, the resolution of the measured acceleration $a$ is

$$
\begin{aligned}
\delta a & =\left|\frac{d O_{1}}{d a}\right|^{-1} \Delta O_{1} \\
& =\left|2 J_{0}(2 \beta \sin \phi) J_{1}(2 \beta \sin \phi) 2 \beta \cos \phi\right|^{-1} \frac{\omega^{2}}{k} \Delta O_{1},
\end{aligned}
$$

where $\Delta O_{1}$ is the experimental uncertainty associated with the observable. The resolution is maximum, i.e., $\delta a$ is minimum, when the lattice position is such that $\phi$ is close to an integer multiple of $\pi$. In this case, since the function $\left|4 J_{0}(2 \beta \sin \phi) J_{1}(2 \beta \sin \phi) \cos \phi\right|$ takes a maximum value approximately equal to 1.2 , weakly dependent on $\beta$ for $\beta>1$, we have

$$
\delta a \approx \frac{\omega^{2}}{k} \frac{1}{\beta} \Delta O_{1}
$$

Therefore, the resolution increases with $\beta$, that is proportional to the number of momentum components significantly populated, showing the benefit of multimode interference. This is consistent with the analysis based on the Fisher information and the Cramér-Rao bound $[19,26]$. Experimentally, the $O_{1}$ peak can be centered at any chosen value of acceleration by controlling the position of the minima of the lattice, extending the dynamic range of this kind of interferometer for the measurements of small forces around $a=0$.

As an alternative to $O_{1}$, we can fit the measured values of the fractional populations at the interferometer output with the squared Bessel functions, having the phase $\phi$ as the single fit parameter. This approach lacks a clear analogy with multiplebeam optical interferometers, but has the advantage of using all populations on the same footing. From a practical point of view we verified that the two approaches yield the same sensitivity. (a)

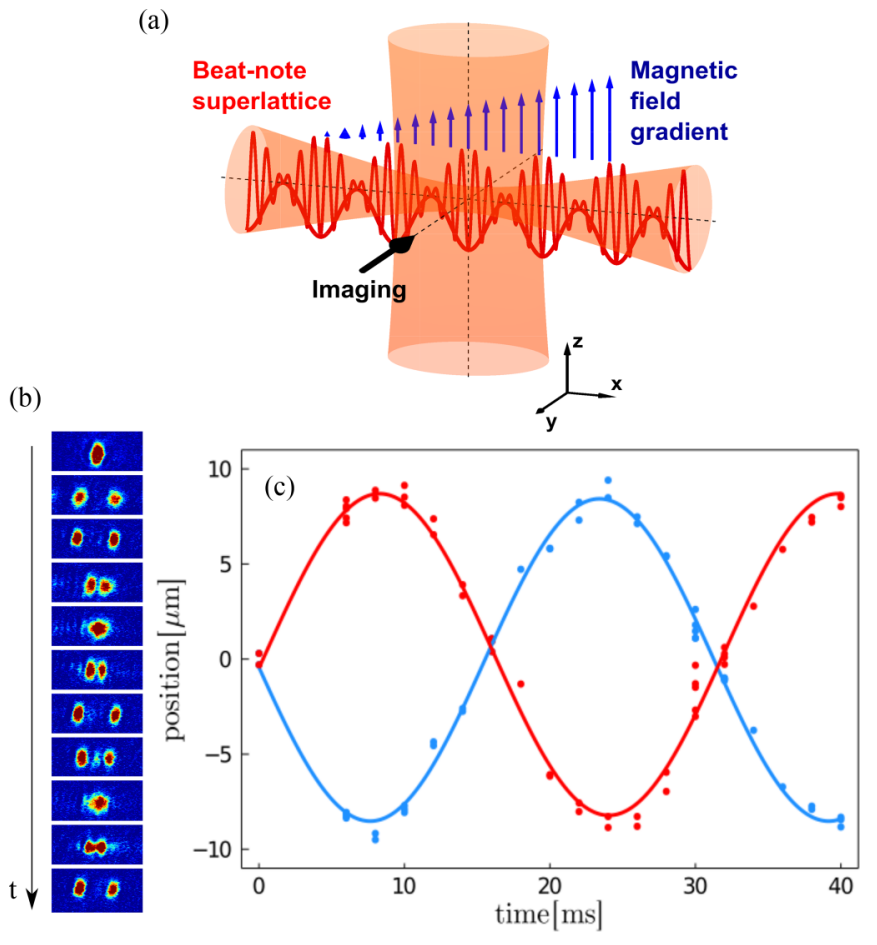

FIG. 2. (a) Sketch of the optical and magnetic potentials applied on the atoms. (b) In situ absorption images of the diffracted orders $m= \pm 1$ during a complete oscillation; (c) corresponding positions as a function of time. Plot data are the outcome of individual measurements; we observe two sinusoidal oscillations with average frequency of $\omega=2 \pi \times 31.7(0.8) \mathrm{Hz}$ and amplitude of 8.6(0.6) $\mu \mathrm{m}$ obtained from the displayed fit.

Finally, for the following it is useful to derive also the output wave function for an interferometer where the two KD pulses are separated by a full-period evolution:

$$
\psi_{F}(x)=\psi_{0}(x) \sum_{m} J_{m}(2 \beta \cos \delta) e^{-i m k x} e^{-i m \phi},
$$

showing that the populations of the momentum components in this full-period interferometer are sensitive only to the relative displacement of the two KD pulses, i.e., to $\delta$ and not to $\phi$. We will exploit this property to calibrate the displacement of the lattice and investigate its stability.

\section{EXPERIMENTAL SETUP}

In order to realize the interferometer, we use a BoseEinstein condensate of $10^{4}{ }^{39} \mathrm{~K}$ atoms in the $\left|f=1, m_{f}=1\right\rangle$ state, that features a broad Feshbach resonance around $400 \mathrm{G}$ [27]. Setting the magnetic field to $B=350.5(0.5) \mathrm{G}$ effectively cancels the interatomic interactions since the corresponding $s$-wave scattering length is $|a|<0.05 a_{0}, a_{0}$ being the Bohr radius.

We prepare the BEC in a crossed dipole trap created by two red-detuned laser beams, as sketched in Fig. 2(a): with a waist of $17 \mu \mathrm{m}$, the horizontal beam provides a tight radial confinement, along $y$ and $z$, of $\omega_{r} \sim 2 \pi \times 200 \mathrm{~Hz}$, while the vertical beam provides the longitudinal harmonic potential, along $x$, with $\omega=2 \pi \times(31.7 \pm 0.8) \mathrm{Hz}$. The comparatively large waist of the vertical beam $(100 \mu \mathrm{m})$ ensures that the 

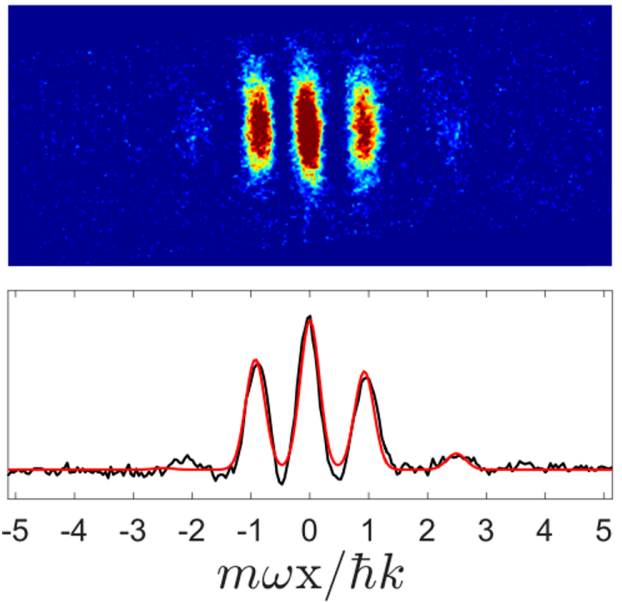
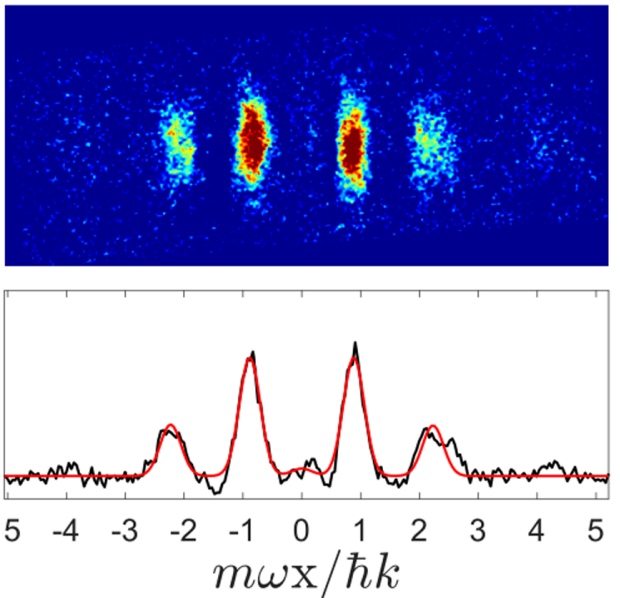

FIG. 3. Absorption image (top) of the momentum distribution and corresponding integrated density profile together with the multiGaussian fit (bottom) after the first KD pulse (left) and after the second KD pulse (right) for a time interval between the two pulses equal to half period, i.e., approximately $16 \mathrm{~ms}$. In both cases a quarter period of in-trap evolution occurs between the last KD pulse and imaging.

deviation from the harmonicity of the potential is below $1 \%$ at a distance of $10 \mu \mathrm{m}$ from the center.

The beat-note superlattice potential along $x$ is generated by overlapping two standing waves $V_{1} \cos ^{2} k_{1} x+$ $V_{2} \cos ^{2} k_{2} x$ with wave vectors $k_{1}=2 \pi / 1.013 \mu \mathrm{m}^{-1}$ and $k_{2}=$ $2 \pi / 1.12 \mu \mathrm{m}^{-1}$, and $V_{1}, V_{2}$ are the lattice depths. For $V_{1}, V_{2}$ of the order of a recoil energy the BEC experiences an effective lattice potential:

$$
V_{\text {eff }}(x)=V_{e 0}[1+\sin (k x+\phi)] / 2 \text {, }
$$

with $V_{e 0}=V_{1} V_{2} M / \hbar^{2}\left(k_{1}+k_{2}\right)^{2}$, where $M$ is the atomic mass, the spatial period $2 \pi / k=\pi /\left(k_{1}-k_{2}\right)$ equals $5.3 \mu \mathrm{m}$, and the phase $\phi$ depends on the relative phase between the two combined lattices $[23,28]$. Both lasers are frequency locked to the same optical reference cavity with a relative stability of $\sim 10 \mathrm{kHz}$, via sideband-locking that allows us to tune $\phi$ dynamically by adjusting the radio frequency of one sideband [29]. Additionally, to investigate the effect of an external force along the $x$ direction we apply a magnetic field gradient, corresponding to an acceleration up to $10^{-3} \mathrm{~g}$, that displaces the minimum of the resultant harmonic potential in a region of few microns.

In order to measure the longitudinal frequency and confirm that the potential is harmonic, at $t=0$ we shine a pulse of the optical lattice with an effective depth $V_{\mathrm{e} 0} \simeq 30 E_{R}$ for $120 \mu \mathrm{s}$, where $E_{R}=\hbar^{2} k^{2} / 2 M=k_{B} \times 8.7 \mathrm{nK}$. The pulse is long enough to completely deplete the $m=0$ component. We record the images of the two components $m= \pm 1$ via absorption imaging (line of sight along the $y$ direction) and we report their position as a function of time: in Figs. 2(b) and 2(c), both components display clear sinusoidal oscillations with an amplitude of 8.6(0.6) $\mu \mathrm{m}$, in reasonable agreement with the expected value $\hbar k / M \omega=9.7(0.2) \mu \mathrm{m}$. Then, we set the KD pulse duration to $80 \mu$ s in order to transfer almost $50 \%$ of the atoms in the $m= \pm 1$ orders and we shine the second KD pulse after a half (or full) period to complete the interferometric sequence. We then image the different momentum orders after allowing an additional quarter oscillation in the trap to maximize their spatial separation. We get the atom number in each components, $N_{m}$, fitting the profiles with a multi-Gaussian function, shown in Fig. 3.

A peculiarity of the beat-note superlattice is represented by the fact that the number of interfering atoms $N$ is coupled to $\beta$. Indeed, beyond the effective potential approximation, the KD pulses diffract atoms also at momentum components associated with the two fundamental optical lattices, i.e., at integer multiples of $2 \hbar k_{1,2}$ [23], and the atoms of these components are effectively lost for the purpose of the interferometer: due to their large momenta, they are driven in the anharmonic region of the ODT (if not outside). In practice, increasing $\beta$ reduces $N$, the total number of atoms contributing to the interferometer signal; for this reason we work with $\beta<2$ [28].

\section{CALIBRATION OF THE INTERFEROMETER}

As shown in Fig. 3 and in agreement with Eq. (1), the final momentum distribution is an even function of $m$, independently of the phases $\phi$ and $\delta$, for both the half- and the full-period interferometer; thus, $\langle p\rangle=0$. However, this symmetry breaks if the time separation of the two KD pulses is not exactly half period, i.e., $\Delta t=(1 / 2+\epsilon) T$. In this case, the $m$ th momentum component acquires an extra phase, $\exp \left(i \epsilon 2 \pi m^{2} E_{R} / \hbar \omega\right)$, given by the classical action in the time interval between the KD pulses. We exploit this sensitivity to precisely determine the oscillation period, using the fullperiod interferometer, that is inherently more stable, since it is insensitive to the relative displacement between the trap and the lattice (and thus to external forces). We identify the period as the time separation $\Delta t$ that yields a symmetric momentum distribution; indeed Fig. 4 shows that measuring the average momentum $\langle p\rangle$ as a function of $\Delta t$ allows us to find the oscillation period with a precision of $10^{-4}$, a factor 27 more accurate than what was possible by measuring the oscillation of the spatial displacement of the wave packets, shown in Fig. 2. Then this value is used to set the time separation equal to $T / 2$, in the half-period interferometer which is sensitive to external forces. 


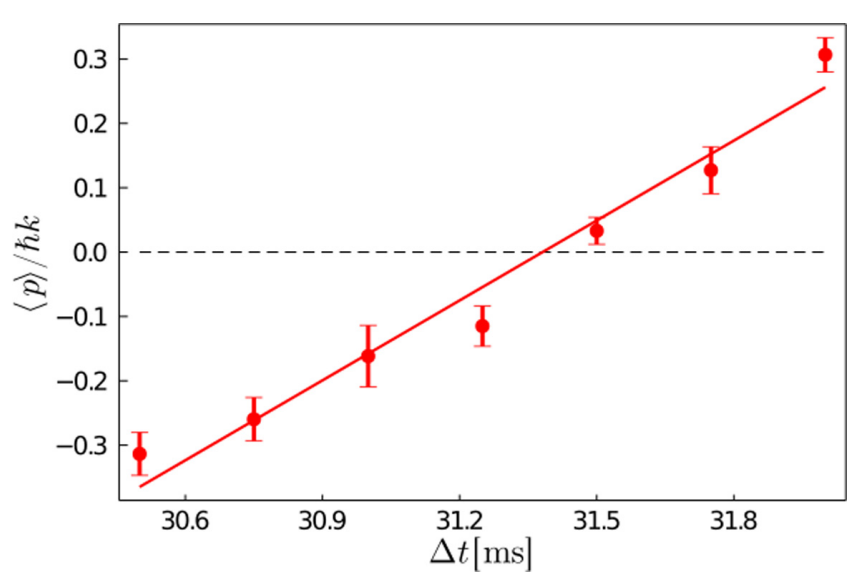

FIG. 4. Average momentum at the output of the full-period interferometer as a function of the time separation between the two KD pulses, $\Delta t$. The fit parameter is the harmonic period, identified as the zero-crossing time: $T=(31.38 \pm 0.03) \mathrm{ms}$. The error bars indicate the standard error on the mean of repeated measurements $(N \sim 5)$.

In addition, with the full-period interferometer we investigate the stability of the relative position of the lattice between the two KD pulses. Equation (4) shows that, at the output of the full-period interferometer, the fraction of population at zero momentum equals $J_{0}^{2}(2 \beta \cos \delta)$. First, we verify this relation thanks to the dynamical control of the lattice position obtained by frequency-shifting one of the two standing waves. We frequency-shift the $k_{2}$ standing wave by $\delta f_{2}=c \delta k_{2} /(2 \pi)$, which displaces the beat-note superlattice by $\delta x_{o}=L \delta k_{2} /\left(k_{1}-k_{2}\right)$, where $L$ is the distance of the atoms from the retroreflecting mirror. We have calibrated the displacement $\delta x_{o}$ by in situ imaging of the position of atoms trapped in the lattice minima, and we obtained $\delta x_{o} / \delta f_{2}=$ $(10 \pm 1) \mathrm{nm} / \mathrm{MHz}$. Thus, during the full-period oscillation we displace the lattice potential by applying the frequency shift $\delta f_{2}$, which modifies $\phi_{2}$ by $k \delta x_{o}$, and we measure $O_{1}$ as a function of $\delta f_{2}$ : as shown in Fig. 5 data are well in agreement with the predicted behavior. Since repeated measurements with the full-period, unshifted interferometer show that the $O_{1}$ is constant within 0.017 (standard deviation; see Fig. 6), we conclude that the relative displacement between the two KD pulses, over the timescale of one oscillation period, i.e., approximately $30 \mathrm{~ms}$, is bounded to be below $0.2 \mu \mathrm{m}$.

\section{MEASUREMENT OF THE APPLIED FORCE}

With these results in hand, we proceed to measure a real force or acceleration. Since the ${ }^{39} \mathrm{~K}$ atoms feature a magnetic moment approximately equal to $0.95 \mu_{B}$ ( $\mu_{B}$ being the Bohr magneton) around $350 \mathrm{G}$, we impart a force along the direction of the lattice by applying an external magnetic field gradient produced with a pair of coils in anti-Helmholtz configuration and a magnitude of about $3 \times 10^{-2} \mathrm{G} / \mathrm{cm} / \mathrm{A}$. The resulting uniform force induces a displacement of the harmonic trap proportional to the coil current, which affects the momentum populations at the interferometer output: the displacement-versus-current conversion has been separately calibrated to be $\eta=(1.08 \pm 0.13) \mu \mathrm{m} / \mathrm{A}$, via the in situ position of atoms trapped in the ODT.

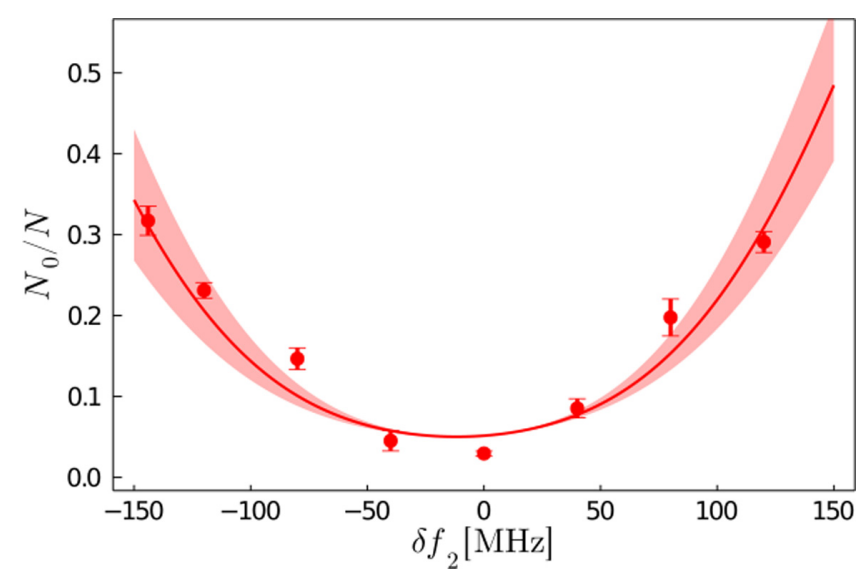

FIG. 5. Fraction of atoms in $m=0$ momentum component, $O_{1}=N_{0} / N$, at the output of the full-period interferometer as a function of the frequency shift of $k_{2}$ standing wave during the second KD pulse. The shaded band is the uncertainty due to the calibration of the beat-note superlattice displacement versus the frequency shift, i.e., $(10 \pm 1) \mathrm{nm} / \mathrm{MHz}$; the error bars indicate the standard error on the mean.

In Fig. 6 we report the measured $O_{1}$ observable as a function of the coil current, with error bars corresponding to the statistical standard deviation for typically 5 repetitions of each data point. The peak identifying the configuration of zero force is not located at $I=0$ due to the presence in our setup of a spurious magnetic field gradient that is canceled by the gradient applied with a current $I=2.8 \mathrm{~A}$. The error bar of $\Delta O_{1}=0.1$, at the maximum slope of the fit curve, yields a resolution of $\Delta I=0.05 \mathrm{~A}$, from which we obtain $\Delta a=\eta \Delta I \omega^{2}=2.19(0.26) \times 10^{-4} \mathrm{~g}$, where the uncertainty is actually dominated by the systematic error due to calibration factor $\eta$.

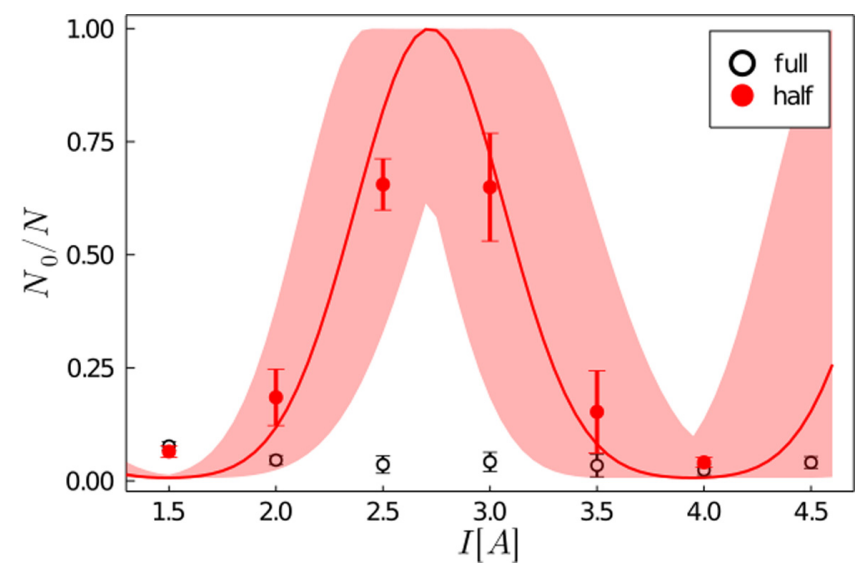

FIG. 6. Fraction of atoms in $m=0$ momentum component at the interferometer output as a function of the magnetic-gradient current, with a trap frequency $\omega=2 \pi \times 31.7 \mathrm{~Hz}$ for the half-period (red, solid points) and full-period (black, open) interferometer. The red line shows the theoretical prediction from Eq. (1), with $\beta=1.12$ and an offset phase as fit parameters; the shaded band is the uncertainty of the displacement-versus-current calibration; the error bars represent the standard error on the mean. 


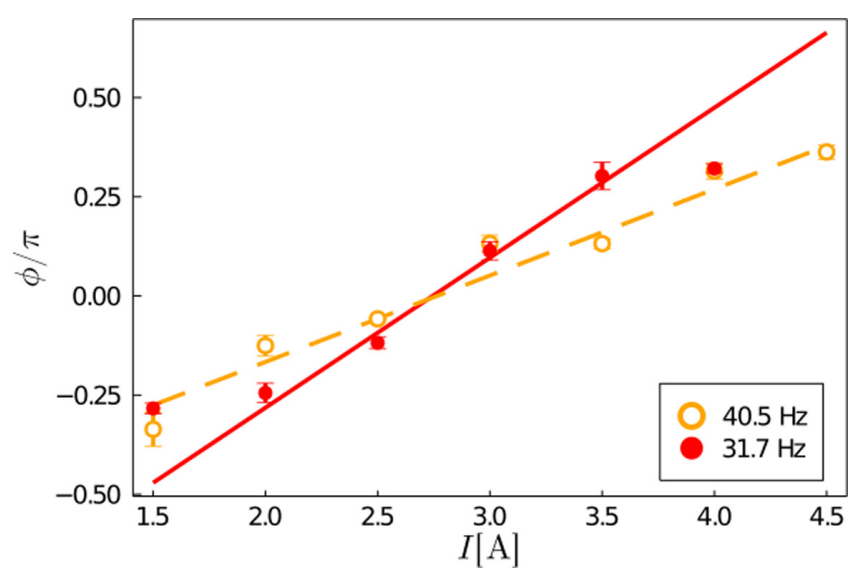

FIG. 7. Interferometric phase $\phi$ derived from fitting the momentum fractional populations of momentum components with $J_{m}^{2}(2 \beta \sin \phi)$, for harmonic frequencies equal to $31.7 \mathrm{~Hz}$ (red, solid points) and $40.5 \mathrm{~Hz}$ (orange, open); lines are linear fits with slopes $(1.18 \pm 0.09) \mathrm{rad} / \mathrm{A}$ (red, solid, excluding extreme points) and $(0.68 \pm 0.08) \mathrm{rad} / \mathrm{A}$ (orange, dashed). The error bars represent the standard error on the mean.

We also assess the acceleration resolution in a complementary manner, i.e., by extracting the interferometric phase $\phi$ from a fit of the populations of all momentum components, for each value of the applied force. This phase is expected to depend linearly on the force, hence on the current: indeed this is what we observe in Fig. 7. From the average error bar of these data, $\langle\Delta \phi\rangle=0.06$, we obtain the acceleration sensitivity $\Delta a=\left(\omega^{2} / k\right)\langle\Delta \phi\rangle=2.2 \times 10^{-4} g$, consistent with the one above.

These values must be compared to the Cramér-Rao bound reported in [19], i.e., $\Delta a_{C R}=\left(\omega^{2} / \beta k\right) 1 /(\sqrt{8 p N})$, where $N$ is the number of atoms, and $p$ is the number of repeated measurements. In our experiment, $N \simeq 5 \times 10^{3}$ and $p$ is typically 4 , thus $1 / \sqrt{8 p N}=2.5 \times 10^{-3}$, which is approximately a factor 40 smaller than our experimental $\Delta O_{1}$. In other words, our resolution does not reach the standard quantum limit corresponding to $\Delta a_{C R}=4 \times 10^{-6} \mathrm{~g}$. While a more systematic investigation is needed, we believe that the main cause for the suboptimal performance is the pointing instability of the ODT beams. Indeed, any technical displacement of the trap minimum is indistinguishable from those induced by external accelerations; the interferometric sequence lasts only $16 \mathrm{~ms}$ but the sample preparation (dead time) takes approximately half a minute, and slow drifts of the ODT beams occur over this timescale. The measured sensitivity is equivalent to a displacement of $0.2 \mu \mathrm{m}$, reasonably of the same order of the slow drifts of the ODT position.

The above discussion shows that lowering the harmonic frequency improves the resolution. However, in our setup we can only marginally increase-and not decrease- the trapping frequency, to keep under control the anharmonicity of the potential [28]. Thus, we repeated the measurement with $\omega=2 \pi \times(40.5 \pm 0.5) \mathrm{Hz}$ and, as expected, the measured interferometric phase is less sensitive to the applied force (see Fig. 7).

\section{CONCLUSIONS}

In conclusion, we have performed a proof-of-principle demonstration of a multimode interferometer in a harmonic trap based on KD diffraction pulses. We have shown that external accelerations are detected from the displacement induced on the harmonic trap with respect to the KD lattice. With a relatively low number of atoms $N \simeq 5 \times 10^{3}$ and a harmonic frequency of approximately $32 \mathrm{~Hz}$, we showed a sensitivity $\delta a \simeq 2 \times 10^{-4} \mathrm{~g}$. Our result is a factor 40 away from the Cramér-Rao bound due to instabilities of the position of the harmonic potential with respect to the lattice.

For a better insight on the potential performance of the KD interferometer, we rewrite Eq. (3) as

$$
\frac{\delta a}{a} \simeq \frac{\hbar \omega}{M a A} \Delta O_{1}
$$

to show that the relative sensitivity is inversely proportional to the potential energy difference of the external force at distances equal to the oscillation amplitude of the atoms with the largest momentum, $A=\beta \hbar k /(M \omega)$. This expression shows that our method represents a simple way to enhance the sensitivity of a trapped atom interferometer by enlarging the spatial separation between the modes involved. In particular this is done by (i) reducing the harmonic trapping frequency, and (ii) increasing the number of momentum components with KD pulses of enhanced intensity and/or duration [30].

In the future it will be interesting to explore the performance of the sensor using a harmonic magnetic confinement along the direction of the lattice, that is more stable in position than the ODT and features a harmonic region much larger. For example, using $10^{5}$ atoms in a magnetic trap with frequency $\approx 1 \mathrm{~Hz}$ and with oscillation amplitudes of $\approx 1 \mathrm{~mm}$, we expect an improvement of the sensitivity by several orders of magnitude, up to $\sim 10^{-8} \mathrm{~g}$. In a magnetic trap, the inhomogeneous magnetic field experienced by the atoms along the oscillation is a concern since it changes the interatomic interaction strength: for ${ }^{39} \mathrm{~K}$ atoms, the above combination of magnetic confinement and oscillation amplitudes implies that the variation of magnetic field is $\approx 10 \mathrm{mG}$, corresponding to a negligible variation of the scattering length $\approx 0.006 a_{0}$. Interestingly, the KD interferometer could be also implemented with spin-polarized fermionic atoms that, at low temperature, are naturally noninteracting, provided that the KD lattice spacing is chosen smaller than the coherence length of the atomic sample [31].

\section{ACKNOWLEDGMENTS}

We acknowledge fruitful discussions with A. Smerzi and we thank M. Prevedelli for a critical reading of the manuscript. This work was supported by the projects TAIOL of QuantERA ERA-NET Cofund in Quantum Technologies (Grant Agreement No. 731473) and QOMBS of FET Flagship on Quantum Technologies (Grant Agreement No. 820419), implemented within the European Union Horizon 2020 Programme. 
[1] A. Peters, K. Y. Chung, and S. Chu, Measurement of gravitational acceleration by dropping atoms, Nature (London) $\mathbf{4 0 0}$, 849 (1999)

[2] A. D. Cronin, J. Schmiedmayer, and D. E. Pritchard, Optics and interferometry with atoms and molecules, Rev. Mod. Phys. 81, 1051 (2009).

[3] T. L. Gustavson, P. Bouyer, and M. A. Kasevich, Precision Rotation Measurements with an Atom Interferometer Gyroscope, Phys. Rev. Lett. 78, 2046 (1997).

[4] P. Cladé, E. de Mirandes, M. Cadoret, S. Guellati-Khélifa, C. Schwob, F. Nez, L. Julien, and F. Biraben, Determination of the Fine Structure Constant Based on Bloch Oscillations of Ultracold Atoms in a Vertical Optical Lattice, Phys. Rev. Lett. 96, 033001 (2006).

[5] G. Rosi, F. Sorrentino, L. Cacciapuoti, M. Prevedelli, and G. Tino, Precision measurement of the Newtonian gravitational constant using cold atoms, Nature (London) 510, 518 (2014).

[6] R. H. Parker, C. Yu, W. Zhong, B. Estey, and H. Müller, Measurement of the fine-structure constant as a test of the standard model, Science 360, 191 (2018).

[7] N. Gaaloul, H. Ahlers, T. Schulze, Y. Singh, S. Seidel, W. Herr, W. Ertmer, E. Rasel et al., Quantum tests of the equivalence principle with atom interferometry, Acta Astronaut. 67, 1059 (2010).

[8] P. Asenbaum, C. Overstreet, M. Kim, J. Curti, and M. A. Kasevich, Atom-Interferometric Test of the Equivalence Principle at the $10^{-12}$ Level, Phys. Rev. Lett. 125, 191101 (2020).

[9] T. Berrada, S. van Frank, R. Bücker, T. Schumm, J.-F. Schaff, and J. Schmiedmayer, Integrated Mach-Zehnder interferometer for Bose-Einstein condensates, Nat. Commun. 4, 2077 (2013).

[10] T. G. Tiecke, M. Kemmann, C. Buggle, I. Shvarchuck, W. von Klitzing, and J. T. M. Walraven, Bose-Einstein condensation in a magnetic double-well potential, J. Opt. B: Quantum Semiclassical Opt. 5, S119 (2003).

[11] S. Pandey, H. Mas, G. Drougakis, P. Thekkeppatt, V. Bolpasi, G. Vasilakis, K. Poulios, and W. von Klitzing, Hypersonic BoseEinstein condensates in accelerator rings, Nature (London) 570, 205 (2019).

[12] T. Schumm, S. Hofferberth, L. M. Andersson, S. Wildermuth, S. Groth, I. Bar-Joseph, J. Schmiedmayer, and P. Krüger, Matterwave interferometry in a double well on an atom chip, Nat. Phys. 1, 57 (2005).

[13] M. Albiez, R. Gati, J. Fölling, S. Hunsmann, M. Cristiani, and M. K. Oberthaler, Direct Observation of Tunneling and Nonlinear Self-Trapping in a Single Bosonic Josephson Junction, Phys. Rev. Lett. 95, 010402 (2005).

[14] G. Spagnolli, G. Semeghini, L. Masi, G. Ferioli, A. Trenkwalder, S. Coop, M. Landini, L. Pezzé, G. Modugno, M. Inguscio, A. Smerzi, and M. Fattori, Crossing Over from Attractive to Repulsive Interactions in a Tunneling Bosonic Josephson Junction, Phys. Rev. Lett. 118, 230403 (2017).

[15] B. Pelle, A. Hilico, G. Tackmann, Q. Beaufils, and F. Pereira dos Santos, State-labeling Wannier-Stark atomic interferometers, Phys. Rev. A 87, 023601 (2013).

[16] R. Charrière, M. Cadoret, N. Zahzam, Y. Bidel, and A. Bresson, Local gravity measurement with the combination of atom in- terferometry and Bloch oscillations, Phys. Rev. A 85, 013639 (2012).

[17] D. S. Naik, G. Kuyumjyan, D. Pandey, P. Bouyer, and A. Bertoldi, Bose-Einstein condensate array in a malleable optical trap formed in a traveling wave cavity, Quantum Sci. Technol. 3, 045009 (2018).

[18] I. Nałcz, L. Masi, G. Ferioli, T. Petrucciani, M. Fattori, and J. Chwedeńczuk, Sensitivity bounds of a spatial Bloch-oscillation atom interferometer, Phys. Rev. A 102, 033318 (2020).

[19] W. D. Li, T. He, and A. Smerzi, Multimode Kapitza-Dirac Interferometry with Trapped Cold Atoms, Phys. Rev. Lett. 113, 023003 (2014).

[20] Y.-J. Wang, D. Z. Anderson, V. M. Bright, E. A. Cornell, Q. Diot, T. Kishimoto, M. Prentiss, R. A. Saravanan, S. R. Segal, and $\mathrm{S}$. Wu, Atom Michelson Interferometer on a Chip Using a Bose-Einstein Condensate, Phys. Rev. Lett. 94, 090405 (2005).

[21] R. E. Sapiro, R. Zhang, and G. Raithel, Atom interferometry using Kapitza-Dirac scattering in a magnetic trap, Phys. Rev. A 79, 043630 (2009).

[22] A. Burchianti, C. D'Errico, L. Marconi, F. Minardi, C. Fort, and M. Modugno, Effect of interactions in the interference pattern of Bose-Einstein condensates, Phys. Rev. A 102, 043314 (2020).

[23] L. Masi, T. Petrucciani, G. Ferioli, G. Semeghini, G. Modugno, M. Inguscio, and M. Fattori, Spatial Bloch Oscillations of a Quantum Gas in a "Beat-Note" Superlattice, Phys. Rev. Lett. 127, 020601 (2021).

[24] J. M. Hogan, D. M. S. Johnson, and M. A. Kasevich, Lightpulse atom interferometry, in Proceedings of the International School of Physics "Enrico Fermi" on Atom Optics and Space Physics, edited by E. Arimondo, W. Ertmer, and W. P. Schleich (IOS Press, Amsterdam, 2009), pp. 411-447.

[25] J. H. Huckans, I. B. Spielman, B. L. Tolra, W. D. Phillips, and J. V. Porto, Quantum and classical dynamics of a Bose-Einstein condensate in a large-period optical lattice, Phys. Rev. A 80 , 043609 (2009).

[26] R. Cheng, T. He, W. Li, and A. Smerzi, Theory of a KaptizaDirac interferometer with cold trapped atoms, J. Mod. Phys. 7 , 2043 (2016).

[27] C. D’Errico, M. Zaccanti, M. Fattori, G. Roati, M. Inguscio, G. Modugno, and A. Simoni, Feshbach resonances in ultracold ${ }^{39}$ K, New J. Phys. 9, 223 (2007).

[28] See Supplemental Material at http://link.aps.org/supplemental/ 10.1103/PhysRevResearch.3.043188 for further discussion of the KD diffraction with the beat-note superlattice and the anharmonicity of the trapping potential.

[29] G. Milani, B. Rauf, P. Barbieri, F. Bregolin, M. Pizzocaro, P. Thoumany, F. Levi, and D. Calonico, Multiple wavelength stabilization on a single optical cavity using the offset sideband locking technique, Opt. Lett. 42, 1970 (2017).

[30] In an ODT the oscillations of the largest momentum components are harmonic only if their amplitude is much smaller than the beam's waist $w$, i.e., $\beta \hbar k /(M \omega) \ll w$, which constraints the achievable sensitivity.

[31] G. Roati, E. de Mirandes, F. Ferlaino, H. Ott, G. Modugno, and M. Inguscio, Atom Interferometry with Trapped Fermi Gases, Phys. Rev. Lett. 92, 230402 (2004). 